\title{
The study and teaching of a language cannot be divorced from its social context.
}

\author{
Mustafa Khalid Saleh Al-Rawi \\ MA English Language and Applied Linguistics (ELAL) \\ Assistant Lecturer at the department of English Language Cihan University - Sulaimanyah -Iraq
}

\begin{abstract}
The aim of this study is to briefly discuss the relationship between language and culture and provide some examples of this relation from the perspectives of studying and teaching a language. It is important to understand the relationship between language and culture and to know which is controlling which. This paper somehow leaned towards the positive aspects of this relation and focused on educational systems that are flexible regarding cultural concepts when studying/teaching a language.
\end{abstract}

Key words : Language teaching/learning, language and culture, code-switching, Language identity

\section{INTRODUCTION}

Languageisanabstracttermwhichgenerallymeans"themethodofhumancommunicationitherspokenorwritt en,consistingoftheuseofwordsinastructuredandconventionalwayOxforddictionaryofEnglish,2005).Languageisa meansofcommunicationbetweenopleofthesamegeographicalplacewithouttheregardoflanguageglobalizationandl anguageboundaries.Peopleofthesamegeographicalplaces(countries)usuallyhaveonelanguageconsideredastheirm othertongueandalsotheyhavetheconceptsofculture.ultureisalsoanabstractconcepthasmanydefinitionsintheliteratu rebutingeneral,is:theartsandothermanifestationsofhumanintellectualachievementregardedcollectively(Oxforddic tionaryofEnglish,2005).Fromthisdefinition,onemaytellthatthereisanimplicationofcommunicationwithinthisdefin itionandalthoughonemaythinkthatwithoutlanguage(communication)culturalaspectsandconceptswouldnotbeexist ed,whileothersthinkthatitisculturethatcreateslanguage.Sincethereisanoverlappingbetweenlanguageandcultureand eachgroupofpeopleofthesamegeographicalplacehastheirlanguageandculture, thereisarelationshipbetweenthesetw $\mathrm{o}$ (Holmes,J.2013).Itisimportanttounderstandtherelationshipbetweenlanguageandcultureandtoknow whichiscontr ollingwhich.Thisessaywillbrieflydiscusstherelationshipbetweenlanguageandcultureandprovidesomeexamplesof thisrelationfromtheperspectivesofstudyingandteachinga language.

\section{LANGUAGE STUDY}

varieties and styles:

Asmentionedpreviouslythateachgroupofpeopleaccordingtotheirgeographicalplacehastheirlanguageandc ulture,itisknownthatpeopleofthesamecountrymayhave, aslinguistscalled, 'languagevarieties'andthesevarietiesofthe samelanguageisduetoculturaldifferences(Holmes,J.2013).Sociolinguistsandanthropologistsindicatedthatthestyleo fcommunicationofalanguage(dialects,jargons)ofagroupofpeopleiscomprehendedinaspecificculture(Romaine,S.2 000).So,thesharedlanguagethosegroupusesissomehowshowingtheiridentityi.e.becausetheircultureplayedrolesinch angingthegenerallanguageandmakeitasadialectforthem,thisdialectispartoftheirculturalidentity.Sapir,EandWhorf,B .L.(1956)establishedthehypothesisthat"thefactofthematteristhat 'realworld'istoalargeextentunconsciouslybuiltupo nthelanguagehabitsofthegroupWeseeandhearandotherwiseexperienceverylargelyaswedobecausethelanguagehabit sofourcommunitypredisposecertainchoicesofinterpretation".Thefamousexampleoftheirhypothesisistheuseofthew ord"snow"inthecontextofEskimoandEnglishlanguages. Therefore,languageisusedasasocialtoolandalsoshapeshow people seethe world and that is an example of the relationship between language and culture.

\section{Code-Switching:}

Code-Switching is the turning between two languages or may be more than two, in the case of multilingual orswitchingbetweenthevarietiesofthesamelanguage, inthecaseofmonolingualsandthatishappenedinthesamecontext .So,itistheusageofanumberoflinguisticvarietiessyntacticallyandphonologicallyinonecontext(Romaine,S.2000)Peo pletendtousethecodeswitchingforfunctionalpurposesasMilroy,L.(1987)arguedtheidentificationof 'prestigeforms'h asbeenconsideredintheworkofsociolinguists. Theuseofprestigiousformsissomehowareflectionnotforthelanguagean dgrammarpurposesbutfortheculturalpurposesandaproperexampletothatisJavaneselanguage.Hudson,R.A.(1980),T rudgill,P.(1983),Coulthard,M.(19852 ${ }^{\text {nd }}$ ed.), andHolmes,J.(1992)seemtobeinterestedinthislanguage, becauseithash ierarchicsystemvarietiesandlargenumberoflexicaldifferences.So,forJavanesepeople, thelanguageisdifferentwhethe 
The study and teaching of a language cannot be divorced from its social context.

rtheiraddresseeisofahigherorlowerstatuscomparedwiththem.Itisaschangingthelanguageaccordingtothepersonthat oneistalkingto; thisisthe 'Interpersonal'conceptofHallidaythateachparticularsocialcontexttendstohaveitsown 'Genr e'andpeoplemayswitchtheirinteractionaldiscoursetofitaspecificgenre. Thehierarchyandrankingsystemisoneofthec onceptsofculture,sothisisanotherexampleoftheinterveningofcultureinlanguage.

Case Study:

AnotherexampleoftherelationshipbetweenlanguageandcultureisinthecasestudybyLupyan,G.andDale,R.(2010)Th eyhaveanalysedstatisticallyalargenumberoflanguagesbyusinganumberof'demographicsources'andtheWorldAtlas ofLanguageStructures'.Ithasbeenfoundthatthereisasignificantlinkbetweenthedifficultyofalanguage(morphologica 1ly)andthespeakers'numberofthislanguage;demographically/sociohistorically,thecomplexityofalanguagedepends mostongeographicalubiquitousnessofthislanguageanditsusage(ibid,2010).Theyfoundthatifalanguageisspokenbyal argenumberofpeopleand(is)widelyspread,thenthislanguageislesscomplexmorphologically,duetotheadaptation 'soc ialenvironments'i.e.becauseitiswidelyspreadpeopleismorelikelytosimplifytheirlanguagestructuresinordertoinvolv ediverserangeofspeakersfromdifferentethnicgroupanddifferent 'linguisticbackgrounds'(ibid,2010).Thelinkbetwee nsocialcontextandlanguagechangeissomewhatobvious.Languagesaremorelikelytobelesscomplexthanbefore,beca useofthesocietalpowerofpeopletochangeandalsoculturalaspectsmightbesubjecttochangethroughtime, sotherelatio nshipbetweensocietalpowerofchangeandtheactualchangeinlanguageseemstobedynamic(Lupyan,GandDale,R.201 0).Nettle,D.(1998)andHay,J.\&Bauer,L.(2007)(citedinLupyan,G.andDale,R.2010)alsoarguedthatthereisarelations hipbetweenthestructureofalanguageanditssocialenvironment, andalsohavefoundthatnotonlythesocialenvironment hasrelationstothelanguagestructure,buttothe 'physicalaspectsoftheenvironment' too.Thisrelationshipbetweenphysi calaspectsoftheenvironmentandlanguagestructurehasalsobeenarguedbyMuntoeR.I.etal.(2009citedinLupyan,G.an dDale,R.2010).Inaddition,mentioningagainSapirWhorfexampleoftheword'snow';itsdifferencesbetweenEnglisha ndEskimolanguagesisprobablyaphysicalaspectoftheenvironment, maybebecausetheEskimo'sexposuretosnowisdif ferentofEnglish'sandthatwhatchangedthemeaning.Howeversocialenvironmentismoreprominentintheliterature.Lu pyan,G.andDale,R.(2010)hasdividedlanguagesintotwoprinciplesaccordingtotheiruseandspeakers'number:'exoteri c'whicharethelanguagesthatarewidelyspreadandspokenbyalargenumberofpeoplefromdifferentbackgrounds,forins tance:Englishand 'esoteric'whicharethelanguagesthatarenotspreadandspokenbysmallnumbersofpeople,forinstanc e:Tatar.Therefore,thesocialenvironmenthasmoreeffectontheexotericlanguagesthatisdueto:firstly,thespreadofthese languageswhichmightbedifficulttostayasitisindifferentgeographicalplacesandsecondly,becauseofthelargenumber ofspeakers,especiallynonnatives, the force toward change may be stronger, and the change tend to be always towards the simpler not the complex.

\section{LANGUAGE TEACHING}

Inthestudyof'communicativelanguageteaching'thisistheteachingofalanguagethroughthefocusoncommun ication(interaction),Woods,D.andCakır,H.(2011)arguethattherearetwo 'dimensions' regardingtheteachers'knowled ge;Thefirstdimensionis 'personalimpersonal'andthesecondoneis 'theoreticalpractical'whichmeansthatthereisarelati onshipbetweentheteachers'knowledgeandbeliefs. Therefore,teachersmayfoundthemselvesinthepositionofreflectin gtheir'culture'whileteachingaslongasconsideringbeliefsasignificantpartofculture. Thesetwoteachers'dimensionsar esomehowinterrelated;teachers'obtainedknowledgeandthinkingwhichistheimpersonalsideareappliedtotheirtheoret ical'explicit'knowledgeandwhattheyareactuallydo(implicitly)inpractice(ibid,2011).Asthereisanoverlappinginthep ersonalimpersonaldimensionteachersseemtousetheirbeliefstheoreticallyandpracticallyi.e.their'objectivity'seemsto beaffectedbytheir'subjectivity'(ibid,2011).Itisnotdefinitethatteachersusetheirculturalbeliefswhileteaching. Howev er,inliterature, ithasbeenfoundthatteachersreflecttheirculturalviewsintheircontext.Personally, Ithinkitdependsonthe culturalcontextthatteachersuse. Althoughsometimesthereareteachers'students'beliefsdifferencescommunicativelan guageteachingtendstomakethesedifferencesbeneficialinawaytoknowhowpeoplefromotherculturesthinkandasanap proach,itisanimportantexampletorelatelanguageteachingtocultureAnotherexamplefromtheteachers'perspectivesw hileteachingalanguageistheresearchconductedbyBlanchetCohen,N.andReilly,R.(2013)inQuebecwhichisamulticul turalurbanlocale.Ithasalargenumberofstudentsfromdifferentbackgrounds, whichmakedifficultyineducationsystem s.BlanchetCohen,N.andReilly,R.(2013)highlightedthe 'environmentaleducation'tosolvetheproblemofthislocale;th eproblemiswiththeculturalsignificantdiversityencounteredbytheeducationalsystem."Researchinternationallyshow sthateducationsystemsthatembracelinguisticdiversityaremostbeneficialtostudentlearning,fosteringacademicachiev ement,selfesteemandconfidence"(Cummins,2007Falbo\&deBaessa,2006;Kymlicka,2003citedinBlanchetCohen,N .andReilly,R.2013)However,theclaimofQuebeceducationalsystemistopursueunifiedvaluesthatembracetheFrenchl anguageandculture; andthatwhatputpressureonbothstudentsandteachers(BlanchetCohen,N.andReilly,R.2013).Ibid( 2013)offeredenvironmentaleducationasasolvingtothesepressuresbecauseitcontainsvariablemethodsofteachingthat somehowseparatethedominanceofoneculturalconceptsfromtheeducationalsystem. Althoughthissystemofeducation hasanumberoflimitations, itisapositivesystemprovidessignificantopportunitiesnotonlyinteachingalanguagebutalsot eachingingeneral. 


\section{CONCLUSION}

Tosumup,thisessayhighlightedtheissuethatthereisarelationshipbetweenlanguageandculture, andshowedbri eflysomeexamplesofthisrelationfromtheperspectivesofstudyingandteachingalanguage. Thisrelationseemstobeobvi ous; thecontroversialissueiswhetherthisrelationcouldaffectpositivelyonstudying/teachingalanguageornot.Thispape rissomehowleaningtowardsthepositivesideofthisrelationandfocusedoneducationalsystemsthattendedtobeflexiblere gardingculturalconceptsormaybedonothaveculturaldominancewhenstudying/teachingalanguage. Finally,thesepara tionoflanguageand cultureisslightlydifficult,becauseitissuggestedthatcultureispartoftheidentityofthe language.

\section{REFERENCES}

[1] BlanchetCohen,N.andReilly,R.(2013)Teachers'perspectivesonenvironmentaleducationinmulticulturalcont exts:Towardsculturally-responsiveenvironmentaleducation.TeachingandTeacherEducation, vol.36,pp.1222.

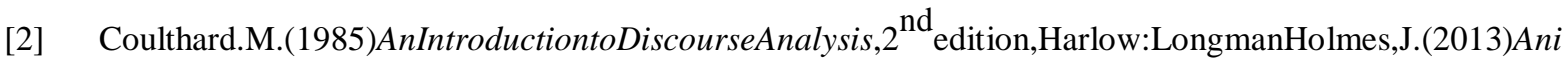
ntroductiontosociolinguistics, $4^{\text {th }}$ edition,London:Routledge.

[3] Hudson,R.A.(1980)Sociolinguistics,Cambridge:CambridgeUniversityPressLupyan,G.andDale,R.(2010)La nguage structureis partlydeterminedbysocial structure.Plosone, vol.5,no.1,pp.1-10.

[4] Milroy,L.(1987)languageandsocialnetworks, $2^{\text {nd }}$ edition,Oxford:BasilBlackwellRomaine,S.(2000)languag einsocietyanintroductiontosociolinguistics, ${ }^{2 \mathrm{nd}}$ edition, Oxford:OxfordUniversityPressTheOxfordEnglishDi ctionary.2nded.2005.App.

[5] Trudgill,P.(1983)OnDialect:SocialandGeographicalPerspectives, Oxford:BasilBlackwellWhorf,B.L.(195 6)'Therelationofhabitualthoughtandbehaviourtolanguage'in Carroll,J.B.(ed.)Language, thought,andreality:SelectedWritingofBenjaminLeeWhorf.MITPressWoods,D.a ndCakır,H.(2011)twodimensionsofteacherknowledge:Thecaseofcommunicativelanguageteaching.System, vol.39, pp. 381-390 